\title{
Computing zero deficiency realizations of kinetic systems
}

\author{
György Lipták ${ }^{a}$, Gábor Szederkényi ${ }^{\mathrm{a}, \mathrm{c}}$, Katalin M. Hangos ${ }^{\mathrm{a}, \mathrm{b}}$ \\ ${ }^{a}$ Process Control Research Group, Systems and Control Laboratory, Institute for \\ Computer Science and Control (MTA SZTAKI), Hungarian Academy of Sciences, \\ Kende u. 13-17, H-1111 Budapest, Hungary \\ ${ }^{\mathrm{b}}$ Department of Electrical Engineering and Information Systems, \\ University of Pannonia, \\ Egyetem u. 10, H-8200 Veszprém, Hungary \\ ${ }^{\mathrm{c}}$ Faculty of Information Technology and Bionics, \\ Pázmány Péter Catholic University, \\ Práter u. 50/a, H-1083 Budapest, Hungary
}

DOI link: http://dx.doi.org/10.1016/j.sysconle.2015.05.001

\begin{abstract}
In the literature, there exist strong results on the qualitative dynamical properties of chemical reaction networks (also called kinetic systems) governed by the mass action law and having zero deficiency. However, it is known that different network structures with different deficiencies may correspond to the same kinetic differential equations. In this paper, an optimization-based approach is presented for the computation of deficiency zero reaction network structures that are linearly conjugate to a given kinetic dynamics. Through establishing an equivalent condition for zero deficiency, the problem is traced back to the solution of an appropriately constructed mixed integer linear programming problem. Furthermore, it is shown that weakly reversible deficiency zero realizations can be determined in polynomial time using standard linear programming. Two examples are given for the illustration of the proposed methods.
\end{abstract}

Key words: nonnegative systems, kinetic systems, optimization, chemical reaction networks, dynamical equivalence

Email addresses: lipgyorgy@scl.sztaki.hu (György Lipták), szederkenyi@itk.ppke.hu (Gábor Szederkényi), hangos@scl.sztaki.hu (Katalin M. Hangos). 


\section{Introduction}

Nonnegative (or positive) dynamical systems, the state variables of which remain nonnegative for nonnegative initial conditions, have important significance in areas such as chemistry, biology, economics, transportation, etc. where the described physical quantities changing in time and/or in space are naturally nonnegative $[11,16]$. An important subclass of nonnegative systems is the family of chemical reaction networks (CRNs, also called kinetic models) rooted in the dynamical description of the concentrations of interacting molecules. Actually, the application potential of kinetic models is much wider than pure chemistry, since nonnegative models from other fields such as disease dynamics, ecology, transportation etc. are often readily in (or can easily be transformed to) kinetic form $[31,24]$. Notable special cases of kinetic models are compartmental systems [16] and Lotka-Volterra systems [30]. Additionally, kinetic systems are the fundamental dynamic model building blocks in systems biology [2].

In (bio)chemical applications, the system parameters (typically the reaction rate coefficients) are uncertain, and often only their order of magnitude is known. Therefore, one of the main subjects of chemical reaction network theory (CRNT) is to give conditions on the qualitative behaviour of kinetic models using mainly the stoichiometry and graph structure of reaction networks $[17,13]$. In [13] and [14], the authors introduce to the study of chemical reaction networks a parameter known as the deficiency, which is a nonnegative integer not depending on the rate coefficients. A classical result of CRNT with clear significance in nonlinear systems theory is the Deficiency Zero Theorem that establishes a robust stability property for deficiency zero reaction networks consisting of strongly connected reaction graph components with a known, parameter-independent logarithmic Lyapunov-function. A promising but technically challenging conjecture not requiring the zero deficiency but only the so-called complex-balanced property for the global stability of a kinetic system is the Global Attractor Conjecture that was proved in [4] for reaction networks having only one graph-component. Furthermore, the Boundedness Conjecture says that any weakly reversible reaction network with mass action kinetics has bounded trajectories (see, e.g. [3]). It is not surprising therefore that the useful properties of kinetic models have raised the interest of control scientists $[5,9]$. In [25], the deficiency zero theorem is revisited and generalized from a control-theoretical point of view by showing that a wide class of CRNs with a linear input structure can be easily stabilized asymptotically. It is shown in [7] that weakly reversible deficiency zero networks are input-to-state stable with respect to the time varying reaction rates as inputs. Moreover, it is possible to construct globally convergent observers for detectable deficiency zero models $[8]$. 
It has been known for a long time, however, that the reaction network representation of a kinetic dynamics is generally not unique, i.e. reaction networks with different structure and/or different set of chemical complexes may represent the same dynamics. This phenomenon is called macro-equivalence [17], confoundability [10] or dynamical equivalence [27], where the possible CRNs corresponding to the same dynamics are called realizations of a kinetic ODE model. The notion of linear conjugacy extends dynamical equivalence by allowing a positive diagonal linear transformation between the states of linearly conjugate realizations [19]. It is known, too, that important model properties such as deficiency, strong connectivity (also called weak reversibility), complex or detailed balance are realization dependent. Therefore, finding dynamically equivalent or linearly conjugate CRN structures with certain required properties can be an interesting and important problem for proving qualitative properties of the model. It was shown that several sub-problems of this class can be successfully solved in the framework of linear and mixed integer linear programming (see, e.g. $[27,28]$ ). In [20] a MILP-based procedure was proposed for finding weakly reversible linearly conjugate realizations of kinetic systems with minimal deficiency. The algorithm was based on the result that for weakly reversible realizations, maximizing the number of reaction graph components minimizes the deficiency. This method uses integer variables for the partitioning of complexes between linkage classes. However, it is known that MILP problems are generally NP-hard and therefore it is often computationally problematic to solve large problems containing integer variables. Moreover, for general non-weakly reversible CRN structures, the basic principle of [20] can not be applied. Therefore, the approach of this paper is different, and our aim is to examine and use the special algebraic consequences of zero deficiency to give a general algorithm for computing such realizations of kinetic systems.

The structure of the paper is the following. In Section 2, the notations used for the representation of kinetic dynamics and linear conjugacy are introduced. Section 3 contains the main result that is an optimization based method for the computation of deficiency zero linearly conjugate kinetic realizations. In Section 4 two illustrative examples are shown, while Section 5 summarizes the contribution of the paper.

\section{Kinetic systems and their realizations}

The basic notions and tools related to reaction kinetic systems and their realizations are briefly summarized in this section with an emphasis on their effect on the structural stability. The following mathematical notations will be used in the paper. $\mathbb{R}_{+}^{n}$ and $\overline{\mathbb{R}}_{+}^{n}$ denote the positive and nonnegative orthant of the $n$-dimensional Euclidean space $\mathbb{R}^{n}$, respectively, and $\mathbf{0}$ denotes 
the zero vector. Similarly, 1 denotes a column vector, all entries of which are 1. For an $n$-dimensional column vector $v, \operatorname{diag}(v)$ is the $n \times n$ diagonal matrix with $v_{1}, \ldots, v_{n}$ in its diagonal. For an arbitrary matrix $M, \operatorname{im}(M)$, $\operatorname{ker}(M)$ and $\operatorname{col}(M)$ denote the image, kernel and the set of columns of $M$, respectively. The element in the $i$ th row and $j$ th column of a matrix $M$ is denoted by $M_{i, j}$ or $[M]_{i, j}$ whenever the latter is more convenient. $V^{\perp}$ and $\operatorname{dim}(V)$ denote the orthogonal complement and dimension of the vector space $V$, respectively, while the sum of vector spaces $V_{1}$ and $V_{2}$ is defined as $V_{1}+V_{2}=$ $\left\{v_{1}+v_{2} \mid v_{1} \in V_{1}, v_{2} \in V_{2}\right\}$. The set of natural numbers (including zero) is denoted by $\mathbb{N}_{0}$. Two matrices $M_{1}, M_{2} \in \mathbb{R}^{n \times m}$ are called structurally equal if the positions of the zero and non-zero elements are the same in $M_{1}$ and $M_{2}$, i.e. $\left[M_{1}\right]_{i, j} \neq 0$ if and only if $\left[M_{2}\right]_{i, j} \neq 0$. Additionally, we will use the following notations known from propositional calculus: ' $\Longrightarrow$ ' and ' $\Longleftrightarrow$ ' denote the 'implies' and 'if and only if' relations between logical expressions having the 'true' or 'false' value.

\subsection{The algebraic structure of kinetic systems}

The general form of dynamic models studied in this paper is the following

$$
\dot{x}=Y \cdot A_{k} \cdot \psi(x),
$$

where $x \in \mathbb{R}^{n}$ is the state vector, $Y \in \mathbb{N}_{0}^{n \times m}, A_{k} \in \mathbb{R}^{m \times m}$ is a special Metzlermatrix defined as:

$$
\left[A_{k}\right]_{i, j}=\left\{\begin{array}{cl}
-\sum_{h=1, h \neq i}^{m} k_{h i} & \text { if } i=j \\
k_{j i} \geq 0 & \text { if } i \neq j
\end{array} .\right.
$$

It is clear from (2) that $A_{k}$ is a matrix with non-positive diagonal and nonnegative off-diagonal elements and zero column sums. Therefore, $A_{k}$ is often called the Kirchhoff-matrix of the system in the theory of kinetic systems. The monomial vector function $\psi: \mathbb{R}^{n} \rightarrow \mathbb{R}^{m}$ is defined as

$$
\psi_{j}(x)=\prod_{i=1}^{n} x_{i}^{Y_{i, j}}, \quad j=1, \ldots, m .
$$

It is easy to show that (1) defines a nonnegative system, i.e. the nonnegative orthant is invariant for its dynamics (see, e.g. [9]). With the notation $M=$ $Y \cdot A_{k}$, the model (1) can be written as

$$
\dot{x}=M \cdot \psi(x) .
$$

A polynomial dynamical system with state vector $x \in \mathbb{R}^{n}$ is called to have the kinetic property (or simply kinetic) if there exist $Y \in \mathbb{N}_{0}^{n \times m}$ and an $m \times m$ 
Kirchhoff matrix $A_{k}$ such that the ODEs of the system can be written in the form of Eq. (1), where $\psi$ is given by (3). The following necessary and sufficient condition for a general polynomial system to be kinetic was given in [18]. Consider a polynomial system written as

$$
\dot{x}=\tilde{M} \cdot \tilde{\psi}(x)
$$

where $\tilde{M} \in \mathbb{R}^{n \times \tilde{m}}$ and $\tilde{\psi}_{j}(x)=\prod_{i=1}^{n} x_{i}^{B_{i, j}}, i=1, \ldots, \tilde{m}$ with $B \in \mathbb{N}_{0}^{n \times \tilde{m}}$. Then, (5) can be written into the form (1), i.e. there exist appropriate matrices $Y$ and $A_{k}$ such that

$$
\tilde{M} \cdot \tilde{\psi}(x)=Y \cdot A_{k} \cdot \psi(x), \quad \forall x \in \overline{\mathbb{R}}_{+}^{n}
$$

if and only if the following condition is fulfilled for $\tilde{M}$ and $B$ :

$$
\text { if } \tilde{M}_{i, j}<0 \text { then } B_{i, j}>0, \text { for } i=1, \ldots, n, j=1, \ldots, \tilde{m} \text {. }
$$

Condition (7) expresses the fact that kinetic systems cannot contain negative cross-effects [31]. In [18], in the framework of a constructive proof, a simple procedure was described to generate a possible $Y, A_{k}$ pair (called the canonical mechanism) such that (6) holds. It has to be noted however, that $Y$ and $A_{k}$ fulfilling (6) for given $\tilde{M}$ and $\tilde{\psi}$ are generally non-unique.

The chemically originated notions of kinetic systems are the following. The species of the system are denoted by $X_{1}, \ldots, X_{n}$, and the concentrations of the species are the state variables of $(1)$, i.e. $x_{i} \geq 0$ for $i=1, \ldots, n$. The structure of kinetic systems is given in terms of its complexes $C_{j}, j=1, \ldots, m$ that are formally the nonnegative integer linear combinations of the species, i.e. $C_{j}=\sum_{i=1}^{n}[Y]_{i, j} X_{i}$ for $j=1, \ldots, m$, and therefore $Y$ is also called the complex composition matrix.

The chemical reactions $C_{i} \rightarrow C_{j}$ where $i \neq j$, with the reaction rate coefficient $k_{i j}>0$, represent the transformation of the complexes into each other with the so called mass action law type reaction rate $r_{i j}$ given by

$$
r_{i j}(x)=k_{i j} \psi_{i}(x)=k_{i j} \prod_{\ell=1}^{n} x_{\ell}^{Y_{\ell, i}}
$$

where $k_{i j}=\left[A_{k}\right]_{j, i}$ as it is written in (2). If $\left[A_{k}\right]_{j, i}=0$ for any $i \neq j$, it means that the reaction $C_{i} \rightarrow C_{j}$ is not present in the system.

\subsubsection{The reaction graph and its incidence matrix}

We can associate a weighted directed graph $G=(\mathcal{V}, \mathcal{E})$, the so called reaction graph to each kinetic system as follows. The elements of the vertex set $\mathcal{V}$ of the reaction graph correspond to the complexes, and the edges in the set $\mathcal{E}$ to the 
reactions. Two complexes $C_{i}$ and $C_{j}$, where $i \neq j$ are connected by a directed edge $\left(C_{i}, C_{j}\right) \in \mathcal{E}$, if a reaction $C_{i} \rightarrow C_{j}$ is present in the kinetic system. Positive weights associated to the edges are the reaction rate coefficients, i.e. the weight corresponding to the directed edge $C_{i} \rightarrow C_{j}$ is $k_{i j}=\left[A_{k}\right]_{j, i}$. The weakly connected components in the reaction graph are called linkage classes, and their number is denoted by $l \geq 1$.

In addition to the Kirchhoff matrix of the system, one can characterize the reaction graph using its incidence matrix $B_{G} \in\{-1,0,1\}^{m \times r}$ where $r$ is the number of reactions. Each reaction in the $\mathrm{CRN}$ is represented by the appropriate column of $B_{G}$ as follows. Let the $\ell$-th reaction in the CRN be $C_{j} \rightarrow C_{i}$ for $1 \leq \ell \leq r$. Then the $\ell$-th column vector of $B_{G}$ is characterized as: $\left[B_{G}\right]_{i \ell}=1$, $\left[B_{G}\right]_{j \ell}=-1$, and $\left[B_{G}\right]_{k \ell}=0$ for $k=1, \ldots, r, k \neq i, j$. It is clear from the above description that the unweighted directed graph structure of a kinetic system can be characterized by the matrix pair $\left(Y, B_{G}\right)$.

\subsection{Properties and dynamics of reaction kinetic systems}

It is possible to utilize certain structural (i.e. parameter-independent) properties of reaction kinetic systems that enable us to effectively analyze the stability of the system. The most important properties of interest from this aspect are deficiency and weak reversibility.

\subsubsection{Deficiency and weak reversibility}

There are several equivalent ways to define the deficiency $\delta$ of a reaction network. We will use the following definition that can be found e.g. in [5]:

$$
\delta=\operatorname{dim}\left(\operatorname{ker}(Y) \cap \operatorname{im}\left(B_{G}\right)\right)
$$

Weak reversibility is the property of the reaction graph only, i.e. it only depends on the structure of the matrix $A_{k}$ or - equivalently - on $B_{G}$. A reaction graph is called weakly reversible if whenever there exists a directed path from $C_{i}$ to $C_{j}$, then there exists a directed path from $C_{j}$ to $C_{i}$. In graph theoretic terms, this means that all components of the reaction graph are strongly connected components. We shall use the fact known from the literature [15] that a CRN is weakly reversible if and only if there exists a vector with strictly positive elements in the kernel of $A_{k}$, i.e. there exists $b \in \mathbb{R}_{+}^{n}$ such that $A_{k} \cdot b=0$. With some abuse of the notions, we will call a Kirchhoff matrix $A_{k}$ weakly reversible if the reaction graph corresponding to $A_{k}$ is weakly reversible.

Here we briefly summarize the Deficiency Zero Theorem published in [14]: (i) If a deficiency zero (not necessarily mass action) network is not weakly 
reversible, then it cannot have a strictly positive equilibrium point. Moreover, the state variables cannot follow a strictly positive cyclic trajectory in this case. (ii) A deficiency zero weakly reversible reaction network with mass action kinetics has precisely one strictly positive equilibrium point in each so-called stoichiometric compatibility class that is at least locally stable with a known logarithmic Lyapunov function, irrespectively of the values of the reaction rate coefficients.

It is visible from the definition that deficiency depends on the graph structure and on the stoichiometry of the reaction network. However, these properties are not encoded into the kinetic differential equations (see, e.g. $[27,20]$ and the next subsection), and different network structures/parametrizations may correspond to the same dynamics. Therefore, finding a deficiency zero structure (either weakly reversible or non-weakly-reversible) reveals valuable information about the qualitative dynamics of the examined kinetic dynamical system.

\subsection{Dynamically equivalent and linearly conjugate realizations}

As it has been mentioned before, the factorization $M=Y \cdot A_{k}$ is generally non-unique (even if $Y$ is fixed), and therefore, generally there exist different reaction structures realizing the same dynamics (dynamical equivalence). Therefore, a matrix pair $\left(Y, A_{k}\right)$ where $Y$ has only nonnegative integer elements and $A_{k}$ is a Kirchhoff matrix is called a dynamically equivalent realization of the kinetic system (4) if $M=Y \cdot A_{k}$. We will assume in the paper that the set of complexes represented by matrix $Y$ is a priori given.

It is known that the kinetic property of a dynamical system is not coordinateindependent and it is preserved only up to the reordering and positive rescaling of the state variables [12]. Therefore, the concept of linear conjugacy was introduced in [19] that allows a positive diagonal transformation between the solutions of two kinetic systems. For our purposes, it is useful to introduce linear conjugacy in a slightly different way than it was described in [19]. For this, let us perform a state transformation on the kinetic model (4) as follows:

$$
\bar{x}(t)=T^{-1} x(t), \forall t,
$$

where $T=\operatorname{diag}(c)$ with $c \in \mathbb{R}_{+}^{n}$. Then the differential equations of the transformed model are given by

$$
\dot{\bar{x}}=T^{-1} \dot{x}=T^{-1} \cdot M \cdot \psi(x)=T^{-1} \cdot M \cdot \psi(T \bar{x})=T^{-1} \cdot M \cdot \psi_{T} \cdot \psi(\bar{x}),
$$

where $\psi_{T}=\operatorname{diag}(\psi(c))$.

Based on the above calculation, a CRN realization $\left(Y, A_{k}^{\prime}\right)$ is called linearly 
conjugate to a kinetic polynomial system of the form (4) if there exists an $n \times n$ positive definite diagonal matrix $T=\operatorname{diag}(c)$ with $c \in \mathbb{R}_{+}^{n}$ such that

$$
Y \cdot A_{k}^{\prime}=T^{-1} \cdot M \cdot \psi_{T}
$$

where $Y \in \mathbb{N}^{n \times m}, \psi_{j}(x)=\prod_{i=1}^{n} x_{i}^{Y_{i j}}$ for $j=1, \ldots, m, \psi_{T}=\operatorname{diag}(\psi(c))$, and $A_{k}^{\prime}$ is an $m \times m$ Kirchhoff matrix. Using the notation $A_{k}=A_{k}^{\prime} \cdot \psi_{T}^{-1}$, we can rewrite (12) as

$$
Y \cdot A_{k}=T^{-1} \cdot M
$$

where $A_{k}$ is a Kirchhoff matrix, too, obtained by scaling the columns of $A_{k}^{\prime}$ by positive scalars. Therefore, $A_{k}$ encodes the same reaction graph structure as $A_{k}^{\prime}$. This implies that the weak reversibility and zero deficiency properties of the CRN realizations $\left(Y, A_{k}\right)$ and $\left(Y, A_{k}^{\prime}\right)$ are equivalent. It is also clear that Eq. (13) is a linear constraint with respect to $A_{k}$ and the diagonal elements of $T^{-1}$. Therefore, instead of $A_{k}^{\prime}$, we will use the scaled matrix $A_{k}$ for representing and computing linearly conjugate realizations.

\section{Computing linearly conjugate zero deficiency realizations}

In this section, two new optimization problems are introduced to compute realizations with zero deficiency. The first one works in the general case and it can be solved as an MILP problem. The second one finds weakly reversible zero deficiency realizations, and it can be traced back to an LP problem.

The basic setup for the computations in both cases will be the following. The known inputs are the coefficient matrix $M$ and the used complex (monomial) set represented by the matrix $Y$. The decision variables to be computed are the scaled Kirchhoff matrix $A_{k}$ of the linearly conjugate realization and the diagonal elements of the inverse transformation matrix $T^{-1}$. Additional auxiliary constants and decision variables (defined later) will also be used to solve the optimization problems.

\subsection{Alternative forms of the definition of zero deficiency}

In this subsection equivalent forms of the zero deficiency condition will be given that can directly be used in the framework of optimization.

The next theorem can be found as Lemma 5.8 in [13]. 
Theorem 1 Let $Y$ and $B_{G}$ be the complex composition matrix and the reaction graph incidence matrix of a kinetic system, respectively. Then $\operatorname{dim}\left(\operatorname{ker}(Y) \cap \operatorname{im}\left(B_{G}\right)\right)=0$ if and only if

$$
\mathbb{R}^{m}=\operatorname{im}\left(Y^{T}\right)+\operatorname{ker}\left(B_{G}^{T}\right) .
$$

The next theorem was originally published as Corollary 4.11 in [13].

Theorem 2 Let $\left(Y, A_{k}\right)$ represent a kinetic system of deficiency zero. Then $\operatorname{ker}\left(A_{k}\right)=\operatorname{ker}\left(Y \cdot A_{k}\right)$.

The following lemma is the immediate consequence of Corollary 4.6 in [13].

Lemma 3 If $A_{k}$ is weakly reversible then $\operatorname{im}\left(B_{G}\right)=\operatorname{im}\left(A_{k}\right)$.

Using the above results, we can state the following theorem.

Theorem 4 Let $\left(Y, A_{k}\right)$ represent a weakly reversible kinetic system. Then $\left(Y, A_{k}\right)$ has zero deficiency if and only if

$$
\operatorname{ker}\left(A_{k}\right)=\operatorname{ker}\left(Y \cdot A_{k}\right)
$$

PROOF. $\Rightarrow$ Let us suppose that $\left(Y, A_{k}\right)$ is a weakly reversible kinetic system with zero deficiency. Then, by Lemma 3 we have that $\operatorname{im}\left(B_{G}\right)=\operatorname{im}\left(A_{k}\right)$. Substituting $A_{k}$ into (9), we obtain that $\operatorname{ker}(Y) \cap \operatorname{im}\left(A_{k}\right)=\mathbf{0}$, from which it follows that $\operatorname{ker}\left(A_{k}\right)=\operatorname{ker}\left(Y \cdot A_{k}\right)$.

$\Leftarrow$ Now, let us assume that $A_{k}$ is weakly reversible and $\operatorname{ker}\left(A_{k}\right)=\operatorname{ker}\left(Y \cdot A_{k}\right)$. The latter implies that

$$
\operatorname{ker}(Y) \cap \operatorname{im}\left(A_{k}\right)=\mathbf{0} .
$$

Due to weak reversibility, we can apply Lemma 3 and $\operatorname{substitute} \operatorname{im}\left(B_{G}\right)$ for $\operatorname{im}\left(A_{k}\right)$ in (16). From this, we obtain that $\operatorname{ker}(Y) \cap \operatorname{im}\left(B_{G}\right)=\mathbf{0}$ that is equivalent to zero deficiency according to Eq. (9).

3.2 Computing linearly conjugate realizations with zero deficiency in the general (not necessarily weakly reversible) case

In order to put the zero deficiency property into the linear programming framework, we are going to reformulate Eq. (14) to the form of linear inequalities.

Eq. (14) is fulfilled if and only if there exist vectors $y^{(\ell)} \in \operatorname{im}\left(Y^{T}\right)$ and $\eta^{(\ell)} \in$ $\operatorname{ker}\left(B_{G}^{T}\right)$, such that an arbitrary basis $\left\{\tilde{y}^{(\ell)}\right\}$ of $\operatorname{ker}(Y)$ can be constructed as

$$
\tilde{y}^{(\ell)}=\eta^{(\ell)}+y^{(\ell)}, \ell=1, \ldots, m-\operatorname{rank}(Y)
$$


Let us use the notation $W=\operatorname{im}\left(Y^{T}\right)+\operatorname{ker}\left(B_{G}^{T}\right)$. Clearly, if (14) holds then $\operatorname{ker}(Y) \subset W$, and therefore any basis of $\operatorname{ker}(Y)$ can be constructed according to Eq. (17). Assume now that an arbitrary basis of $\operatorname{ker}(Y)$ can be written as in Eq. (17). Then $\operatorname{ker}(Y) \subset W$. Since by construction $\operatorname{im}\left(Y^{T}\right) \subset W$, and $\operatorname{im}\left(Y^{T}\right)$ is the orthogonal complement of $\operatorname{ker}(Y)$, we obtain that $\mathbb{R}^{m}=\operatorname{im}\left(Y^{T}\right)+\operatorname{ker}(Y) \subseteq$ $W$. Recall that $W \subseteq \mathbb{R}^{m}$, therefore $W=\mathbb{R}^{m}$.

Since the matrix $Y$ is a priori given and constant, we can easily determine a basis $\left\{\tilde{y}^{(\ell)}\right\}$ of $\operatorname{ker}(Y)$. We can also generate an arbitrary element of $\operatorname{im}\left(Y^{T}\right)$ with the linear combination of the column vectors of $Y^{T}$ as follows:

$$
y^{(\ell)}=Y^{T} \cdot \alpha^{(\ell)},
$$

where $\alpha^{(\ell)} \in \mathbb{R}^{n}$.

Clearly, a vector $\eta^{(\ell)}$ is in the kernel of the matrix $B_{G}^{T}$ if and only if

$$
B_{G}^{T} \cdot \eta^{(\ell)}=\mathbf{0}
$$

Since $B_{G}$ depends on $A_{k}$ (i.e. it depends on the reaction graph of the realization), (19) is nonlinear in the optimization variables. Therefore, we will use the special structure of the matrix $B_{G}$ to convert Eq. (19) to an equivalent form which can be inserted into MILP framework. Using the structure of the incidence matrix $B_{G}$, we can state the following theorem.

Theorem 5 Eq. (19) can be equivalently represented by the following logical expression:

$$
\left[A_{k}\right]_{i, j}>0 \Longrightarrow \eta_{i}^{(\ell)}=\eta_{j}^{(\ell)}, i, j=1, \ldots, m
$$

PROOF. $\Rightarrow$ Assume that $B_{G}^{T} \cdot \eta^{(\ell)}=\mathbf{0}$. Let us take the row $v$ of $B_{G}^{T}$ that corresponds to the directed edge $C_{j} \rightarrow C_{i}$ of the reaction graph. Then $v_{i}=1$, $v_{j}=-1$ and $v_{k}=0$ for $k \neq i, j$. Obviously, $v \cdot \eta^{(\ell)}=\eta_{i}^{(\ell)}-\eta_{j}^{(\ell)}$ which implies (since $\eta^{(\ell)}$ is in the kernel of $B_{G}^{T}$ ) that $\eta_{j}^{(\ell)}=\eta_{i}^{(\ell)}$. We repeat this for each directed edge of the reaction graph and the corresponding row of $B_{G}^{T}$. Then, clearly $\left[A_{k}\right]_{i, j}>0$ implies $\eta_{i}^{(\ell)}=\eta_{j}^{(\ell)}$ using the properties of the Kirchhoff matrix $A_{k}$.

$\Leftarrow$ Assume that $(20)$ is true. Take any $(i, j)$ for which $\left[A_{k}\right]_{i, j}>0$. According to (20) we have that $\eta_{i}^{(\ell)}=\eta_{j}^{(\ell)}$. Let us denote the row vector of $B_{G}^{T}$ corresponding to the directed edge $C_{j} \rightarrow C_{i}$ by $v$. Then $v \cdot \eta^{(\ell)}=0$. Repeating this for each directed edge of the reaction graph, we get that $B_{G}^{T} \cdot \eta^{(\ell)}=\mathbf{0}$.

It is well-known that logical expressions can be expressed with linear inequalities and integer variables (see, e.g. [6]). For this purpose we introduce a binary 
matrix $\Theta \in\{0,1\}^{m \times m}$ such that

$$
\left[A_{k}\right]_{i, j}>0 \Longrightarrow \Theta_{i, j}=1, \forall i, j=1, \ldots, m .
$$

The relation (21) can be expressed using the following equivalent linear inequalities:

$$
\left[A_{k}\right]_{i, j} \leq U_{1} \cdot \Theta_{i, j}, \forall i, j=1, \ldots, m .
$$

where $U_{1} \in \mathbb{R}_{+}$is the upper bound for $\left[A_{k}\right]_{i, j}$.

To ensure (20), we add the following logical expression in addition to (21)

$$
\Theta_{i, j}=1 \Longrightarrow \eta_{i}^{(\ell)}=\eta_{j}^{(\ell)}
$$

Similarly to the above, (23) can also be described with linear inequalities that are the following:

$$
\left|\eta_{i}^{(\ell)}-\eta_{j}^{(\ell)}\right| \leq 2 \cdot U_{2}\left(1-\Theta_{i, j}\right)
$$

where $U_{2} \in \mathbb{R}_{+}$is the upper bound of $\left|\eta_{i}^{(\ell)}\right|$.

Using the calculations described in Subsection 2.3, the sign and columnconservation properties of Kirchhoff matrices, and Eqs. (17), (18), (22), and (24), we can summarize the linear constraints for computing deficiency zero linearly conjugate realizations as follows.

$$
\left\{\begin{array}{l}
\operatorname{diag}\left(\left[d_{1} \ldots d_{n}\right]^{T}\right) \cdot M=Y \cdot A_{k} \\
d_{i}>0, \quad i=1, \ldots, n \\
\mathbf{1}^{T} \cdot A_{k}=\mathbf{0}^{T} \\
{\left[A_{k}\right]_{i j} \geq 0 \quad i, j=1, \ldots, m, i \neq j} \\
\tilde{y}^{(\ell)}=\eta^{(\ell)}+Y^{T} \cdot \alpha^{(\ell)}, \quad \ell=1, \ldots, m-\operatorname{rank}(Y) \\
{\left[A_{k}\right]_{i, j} \leq U_{1} \cdot \Theta_{i, j} \quad i, j=1, \ldots, m} \\
\left|\eta_{i}^{(\ell)}-\eta_{j}^{(\ell)}\right| \leq 2 \cdot U_{2}\left(1-\Theta_{i, j}\right) \quad i, j=1, \ldots, m, \quad \ell=1, \ldots, m-\operatorname{rank}(Y),
\end{array}\right.
$$

The known constants in $(25)$ are $M, Y, \tilde{y}^{(\ell)}$ for $\ell=1, \ldots, m-\operatorname{rank}(Y)$, $U_{1}$ and $U_{2}$. The continuous decision variables are the off-diagonal elements of $A_{k}, d_{i}$ for $i=1, \ldots, n$ (with $T^{-1}=\operatorname{diag}\left(\left[d_{1} \ldots d_{n}\right]^{T}\right)$ ), $\alpha^{(\ell)}$, and $\eta_{i}^{(\ell)}$ for $\ell=1, \ldots, m-\operatorname{rank}(Y)$ and $i=1, \ldots, m$. Additionally, $\Theta_{i, j}$ for $i, j=1, \ldots, m$ are the binary decision variables. The bound $U_{1}$ can be chosen as an arbitrary positive real number (e.g. it can be set to 1 ), because the matrices $T^{-1}$ and $A_{k}$ can be scaled by any positive scalar in Eq. (13). If the constraint set (25) is reported to be infeasible by the applied solver, it is recommended to increase $U_{2}$ as long as the numerical tolerance of the solver permits, to maximize the feasibility domain. 
It is visible that the constraint set (25) is linear in the unknowns, therefore feasible solutions (if exist) can be found in the framework of mixed integer linear programming (MILP). Since the original problem to be solved can be traced back to the feasibility of the constraint set (25), the linear objective function $f_{o b j}$ to be minimized, can be chosen freely, therefore it can be used to ensure additional properties of the computed realizations. A simple choice can be the minimization of the $L^{1}$-norm of the off-diagonal elements of $A_{k}$, i.e.

$$
f_{o b j}=\sum\left[A_{k}\right]_{i j} \text {, for } i, j=1, \ldots, m, i \neq j \text {. }
$$

So-called dense or sparse solutions containing the maximal or minimal number of directed edges in the realization, respectively, can also be computed as it is described in e.g. [27] by modifying (21) to

$$
\left[A_{k}\right]_{i, j}>0 \Longleftrightarrow \Theta_{i, j}=1, \forall i, j=1, \ldots, m
$$

and using the objective function $f_{o b j}= \pm \sum_{i, j=1}^{m} \Theta_{i j}$. But note that in this case, the constraint set (25) changes and becomes more complicated. We remark that the number of complexes for CRNs realizing a given dynamics can also be minimized using the MILP method described in [29] which can be considered as a kind of model reduction. This result is related to [23], where the number of complexes of a complex-balanced CRN is reduced while maintaining the complex balance property and keeping a strong relation between the equilibria of the original and the reduced system.

\subsection{Computing linearly conjugate weakly reversible realizations with zero de- ficiency}

In this subsection we are going to apply Theorems 2 and 4 to compute linearly conjugate weakly reversible realizations with zero deficiency as an LP problem. Let us now recall that a necessary and sufficient condition of weak reversibility is the existence of a strictly positive vector in $\operatorname{ker}\left(A_{k}\right)$. It is easy to see that $\operatorname{ker}(M)=\operatorname{ker}(T \cdot M)$ due to the invertibility of the transformation matrix $T$. If there is no strictly positive vector in $\operatorname{ker}(M)$ then it is trivial that there cannot be a positive vector in $\operatorname{ker}\left(A_{k}\right)$ since $T \cdot M=Y \cdot A_{k}$. Therefore, as a first step of the computation, we have to check that

$$
\exists p \in \mathbb{R}_{+} \text {such that } M \cdot p=\mathbf{0}
$$

is fulfilled.

If (27) holds, then we compute an arbitrary basis of $\operatorname{ker}(M)$ denoted by $\left\{\eta^{(i)}\right\}$, for $i=1, \ldots, m-\operatorname{rank}(M)$. Then, according to Theorem 4, there exists a 
weakly reversible zero deficiency linearly conjugate realization if and only if the constraint set

$$
\left\{\begin{array}{l}
\operatorname{diag}\left(\left[d_{1} \ldots d_{n}\right]^{T}\right) \cdot M=Y \cdot A_{k} \\
d_{i}>0, \quad i=1, \ldots, n \\
\mathbf{1} \cdot A_{k}=\mathbf{0} \\
{\left[A_{k}\right]_{i j} \geq 0 \quad i, j=1, \ldots, m, i \neq j,} \\
A_{k} \cdot \eta^{(i)}=\mathbf{0}, i=1, \ldots, m-\operatorname{rank}(M)
\end{array}\right.
$$

is feasible. The known constants are $M, Y$ and $\eta^{(i)}$ for $i=1, \ldots, m-\operatorname{rank}(M)$. The decision variables are the off-diagonal elements of $A_{k}$ and the elements of the vector $d$. The constraint set (28) is clearly linear and it contains only continuous variables, therefore its feasibility can be decided in polynomial time using linear programming. The objective function $f_{o b j}$ to be minimized can be chosen as an arbitrary linear function of the decision variables here as well. A practical choice for $f_{o b j}$ can be (26) here, too.

If (27) holds, but the constraint set (28) is infeasible, then, according to Theorem 2 , there is no deficiency zero linearly conjugate realization of the kinetic system (4) with the complex set given by $Y$.

The solutions for both (25) and (28) are parametrically not unique because of the possible scaling of Eq. (13) already mentioned in Subsection 3.2. By the structural uniqueness of a CRN realization, we mean the uniqueness of its unweighted reaction graph. Matrix $A_{k}$ of a feasible solution to either (25) or (28) is simply called a feasible $A_{k}$. The CRN realization corresponding to a feasible $A_{k}^{(0)}$ is structurally unique if and only if there is no feasible $A_{k}^{(1)}$ that is structurally not equal to $A_{k}^{(0)}$. This can be easily checked through e.g. a series of optimization steps by adding extra linear constraints to (25) or (28).

\section{Examples}

In the following, two examples will be provided as case studies for the proposed methods. The algorithms were implemented in MATLAB [22] using the YALMIP modelling language [21]. The freely available GLPK package [1] was used to solve the emerging LP and MILP problems. It can be shown for both examples that there is no dynamically equivalent CRN realization for the given dynamics with the complexes defined by matrix $Y$, but there exists a linearly conjugate deficiency zero realization. 
This example illustrates the general MILP approach described in Subsection 3.2. Let us consider a kinetic system of the form (4) characterized by the following complex composition matrix $Y$ and the coefficient matrix $M$ :

$$
Y=\left[\begin{array}{lllll}
0 & 0 & 1 & 0 & 1 \\
2 & 0 & 0 & 1 & 1 \\
0 & 1 & 0 & 0 & 0
\end{array}\right], \quad M=\left[\begin{array}{rrrrr}
0 & 0 & -1 & 4 & -1 \\
-2 & 2 & 0.25 & 0 & 0 \\
1 & -1 & 0 & 0 & 0
\end{array}\right] \text {. }
$$

The differential equations defined by $M$ and $Y$ are given by

$$
\begin{aligned}
& \dot{x}_{1}=-x_{1}+4 x_{2}-x_{1} x_{2} \\
& \dot{x}_{2}=-2 x_{2}^{2}+2 x_{3}+0.25 x_{1} \\
& \dot{x}_{3}=x_{2}^{2}-x_{3} .
\end{aligned}
$$

Now, by using the proposed method for the general (non-weakly-reversible) case we are able to determine a realization $\left(Y, A_{k}^{\prime}\right)$ which has zero deficiency and it is linearly conjugate to the kinetic system $(Y, M)$ with $d_{1}=0.25, d_{2}=$ $1, d_{3}=1$. The resulting $\mathrm{CRN}$ is given by $\left(Y, A_{k}^{\prime}\right)$, where the non-zero offdiagonal elements of the matrix $A_{k}^{\prime}$ are the following: $\left[A_{k}^{\prime}\right]_{2,1}=1,\left[A_{k}^{\prime}\right]_{1,2}=1$, $\left[A_{k}^{\prime}\right]_{4,3}=1,\left[A_{k}^{\prime}\right]_{5,4}=1,\left[A_{k}^{\prime}\right]_{4,5}=1$. The bounds were selected as $U_{1}=U_{2}=10$. The non-weakly-reversible reaction graph of the obtained CRN is shown in Fig. 1. Thus, it follows from the Deficiency Zero Theorem that the corresponding kinetic system (29) has no positive steady states.

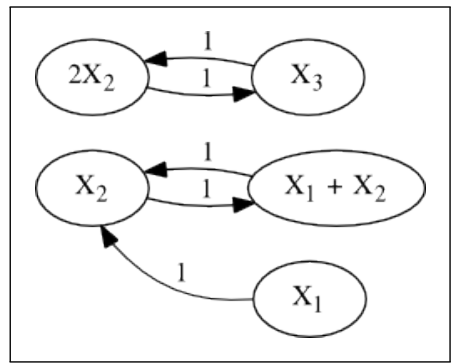

Figure 1. Reaction graph of the obtained realization in Subsection 4.1

\subsection{Computation of a weakly reversible deficiency zero structure}

This example illustrates the LP approach described in Subsection 3.3 and clearly shows that the additional transformation parameters introduced by linear conjugacy might be necessary to find the desired CRN structure. The 
starting kinetic system is given by the matrices $Y$ and $M$ as follows:

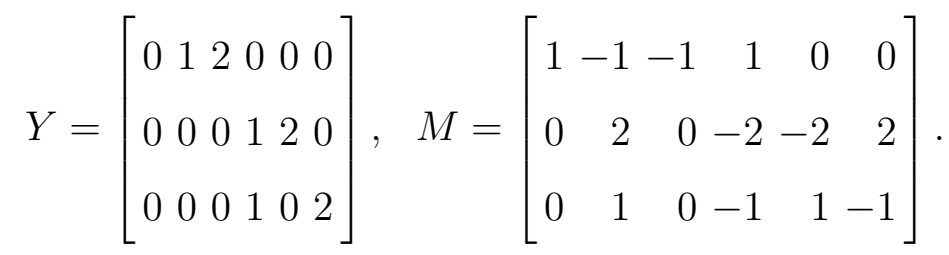

This representation is equivalent to the following differential equations

$$
\begin{aligned}
& \dot{x}_{1}=1-x_{1}-x_{1}^{2}+x_{2} x_{3} \\
& \dot{x}_{2}=2 x_{1}-2 x_{2} x_{3}-2 x_{2}^{2}+2 x_{3}^{2} \\
& \dot{x}_{3}=x_{1}-x_{2} x_{3}+x_{2}^{2}-x_{3}^{2} .
\end{aligned}
$$

It is easy to see that there exists a positive vector in $\operatorname{ker}(M)$, e.g. $\mathbf{1}$, so we can apply the LP method presented in Subsection 3.3. If we try to find a weakly reversible dynamically equivalent deficiency zero realization, we find that the constraints (28) are infeasible with $d_{1}=d_{2}=d_{3}=1$. However, we can find a weakly reversible realization which is linearly conjugate to the original system (30) with the transformation $T=\operatorname{diag}(2,1,2)$ that has zero deficiency. This $\mathrm{CRN}$ is given by $\left(Y, A_{k}^{\prime}\right)$ where the Kirchhoff matrix $A_{k}^{\prime}$ has the following nonzero off-diagonal entries: $\left[A_{k}^{\prime}\right]_{3,1}=1,\left[A_{k}^{\prime}\right]_{4,2}=1,\left[A_{k}^{\prime}\right]_{1,3}=0.25,\left[A_{k}^{\prime}\right]_{2,4}=1$, $\left[A_{k}^{\prime}\right]_{6,5}=1$ and $\left[A_{k}^{\prime}\right]_{5,6}=0.25$. One can see the reversible reaction graph of this network in Fig. 2. Therefore, we obtain from the Deficiency Zero Theorem that the kinetic system (31) has precisely one locally asymptotically stable strictly positive equilibrium point in each stoichiometric compatibility class.

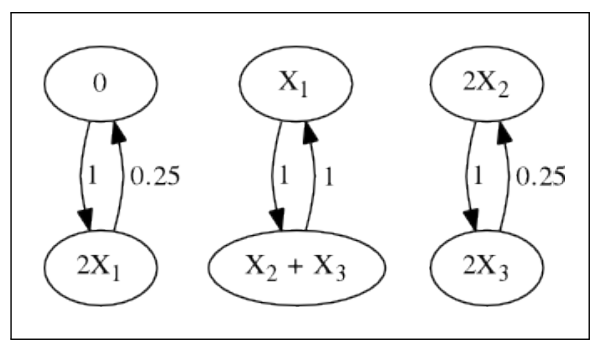

Figure 2. Reaction graph of the obtained realization in Subsection 4.2. This realization is weakly reversible and has zero deficiency.

\section{Conclusions and future work}

\subsection{Summary of contributions}

Optimization-based methods were presented in this paper for the computation of zero deficiency realizations of kinetic polynomial systems. Previously 
known algebraic conditions for zero deficiency and weak reversibility were reformulated to be able to directly include them into the linear optimization framework. It was shown that with a given complex set, weakly reversible deficiency zero linearly conjugate realizations can be found in polynomial time using pure linear programming. It also follows from the computational approach that the existence of deficiency zero weakly reversible linearly conjugate realizations can be decided efficiently even for large kinetic systems. The general non-weakly-reversible deficiency zero case remained a MILP problem in the applied optimization framework.

The developed approach was illustrated through two computational examples, where it could be shown that linearly conjugate realizations indeed represent a wider system class than dynamically equivalent ones in the sense of possible structures. Further work will be focused on the utilization of the results in feedback design for polynomial systems, and thus exploiting kinetic model properties in nonlinear systems theory. The developed algorithms will be included and published in the new version of the CRNreals toolbox [26].

\subsection{Future work: possibilities of treating higher deficiencies}

In this subsection, we briefly examine the treatment of deficiency one in our optimization framework. Using the definition of deficiency in Eq. (9), let us define the subspace $W$ as follows

$$
W=\operatorname{ker}(Y) \cap \operatorname{im}\left(B_{G}\right) .
$$

The orthogonal complement of $W$ is given by

$$
W^{\perp}=\operatorname{im}\left(Y^{T}\right)+\operatorname{ker}\left(B_{G}^{T}\right) .
$$

The deficiency is equal to the dimension of $W$. Therefore, the deficiency is less than or equal to 1 if and only if $\operatorname{dim}(W) \leq 1$. This condition is equivalent to

$$
\mathbb{R}^{m}=W^{\perp}+\operatorname{span}(v),
$$

where $v \in \mathbb{R}^{m}$ is a suitable vector. Now we can give a similar condition to (17). Eq. (34) is fulfilled if and only if there exist vectors $y^{(\ell)} \in \operatorname{im}\left(Y^{T}\right)$, $\eta^{(\ell)} \in \operatorname{ker}\left(B_{G}^{T}\right)$ and $v^{(\ell)} \in \operatorname{span}(v)$, such that an arbitrary basis $\left\{\tilde{y}^{(\ell)}\right\}$ of $\operatorname{ker}(Y)$ can be constructed as

$$
\tilde{y}^{(\ell)}=\eta^{(\ell)}+y^{(\ell)}+v^{(\ell)}, \ell=1, \ldots, m-\operatorname{rank}(Y) .
$$

The construction of vectors $\eta^{(\ell)}$ and $y^{(\ell)}$ has been described in Subsection 3.2. The unknown vectors $v^{(\ell)}$ are parallel to each other by construction, and 
this would introduce a non-linear (quadratic) constraint in the optimization. Therefore, it will be a target of future research to elaborate on the possibilities of computing realizations with given higher deficiencies.

\section{Acknowledgements}

This work was partially supported by the Hungarian Scientific Research Fund under grants no. OTKA NF104706 and K83440. The support of the grant KAP-1.1-14/029 is also acknowledged. The authors thank János Rudan for his support in computing the examples and Bernadett Ács for carefully reading the manuscript. The authors also thank the anonymous reviewers for their constructive and helpful comments.

\section{References}

[1] GLPK - GNU Linear Programming Toolkit.

https://www.glpk.org.

[2] U. Alon. An Introduction to Systems Biology: Design Principles of Biological Circuits. Chapman \& Hall, CRC, 2007.

[3] D. F. Anderson. Boundedness of trajectories for weakly reversible, single linkage class reaction systems. Journal of Mathematical Chemistry, 49:1-16, 2011. DOI: 10.1007/s10910-011-9886-4.

[4] D. F. Anderson. A proof of the Global Attractor Conjecture in the single linkage class case. SIAM Journal on Applied Mathematics, 71:1487-1508, 2011. http://arxiv.org/abs/1101.0761.

[5] D. Angeli. A tutorial on chemical network dynamics. European Journal of Control, 15:398-406, 2009.

[6] A. Bemporad and M. Morari. Control of systems integrating logic, dynamics, and constraints. Automatica, 35:407-427, 1999.

[7] M. Chaves. Input-to-state stability of rate-controlled biochemical networks. SIAM Journal on Control and Optimization, 44:704-727, 2005.

[8] M. Chaves and E. D. Sontag. State-estimators for chemical reaction networks of Feinberg-Horn-Jackson zero deficiency type. European Journal of Control, 8:343-359, 2002.

[9] V. Chellaboina, S. P. Bhat, W. M. Haddad, and D. S. Bernstein. Modeling and analysis of mass-action kinetics - nonnegativity, realizability, reducibility, and semistability. IEEE Control Systems Magazine, 29:60-78, 2009.

[10] G. Craciun and C. Pantea. Identifiability of chemical reaction networks. Journal of Mathematical Chemistry, 44:244-259, 2008. 
[11] L. Farina and S. Rinaldi. Positive Linear Systems: Theory and Applications. Wiley, 2000.

[12] G. Farkas. Kinetic lumping schemes. Chemical Engineering Science, 54:39093915, 1999.

[13] M. Feinberg. Lectures on chemical reaction networks. Notes of lectures given at the Mathematics Research Center, University of Wisconsin, 1979.

[14] M. Feinberg. Chemical reaction network structure and the stability of complex isothermal reactors - I. The deficiency zero and deficiency one theorems. Chemical Engineering Science, 42 (10):2229-2268, 1987.

[15] Martin Feinberg and F.J.M. Horn. Chemical mechanism structure and the coincidence of the stoichiometric and kinetic subspaces. Archive for Rational Mechanics and Analysis, 66(1):83-97, 1977.

[16] W. M. Haddad, VS. Chellaboina, and Q. Hui. Nonnegative and Compartmental Dynamical Systems. Princeton University Press, 2010.

[17] F. Horn and R. Jackson. General mass action kinetics. Archive for Rational Mechanics and Analysis, 47:81-116, 1972.

[18] V. Hárs and J. Tóth. On the inverse problem of reaction kinetics. In M. Farkas and L. Hatvani, editors, Qualitative Theory of Differential Equations, volume 30 of Coll. Math. Soc. J. Bolyai, pages 363-379. North-Holland, Amsterdam, 1981.

[19] M. D. Johnston and D. Siegel. Linear conjugacy of chemical reaction networks. Journal of Mathematical Chemistry, 49:1263-1282, 2011.

[20] M. D. Johnston, D. Siegel, and G. Szederkényi. Computing weakly reversible linearly conjugate chemical reaction networks with minimal deficiency. Mathematical Biosciences, 241:88-98, 2013.

[21] J. Löfberg. YALMIP : A toolbox for modeling and optimization in MATLAB. In Proceedings of the CACSD Conference, Taipei, Taiwan, 2004.

[22] The Math Works, Inc., Natick, MA. Matlab User's Guide, 2000.

[23] S. Rao, A.J. van der Schaft, and B. Jayawardhana. A graph-theoretical approach for the analysis and model reduction of complex-balanced chemical reaction networks. Journal of Mathematical Chemistry, 51:2401-2422, 2013.

[24] N. Samardzija, L. D. Greller, and E. Wassermann. Nonlinear chemical kinetic schemes derived from mechanical and electrical dynamical systems. Journal of Chemical Physics, 90 (4):2296-2304, 1989.

[25] E. Sontag. Structure and stability of certain chemical networks and applications to the kinetic proofreading model of T-cell receptor signal transduction. IEEE Transactions on Automatic Control, 46:1028-1047, 2001.

[26] G. Szederkényi, J. R. Banga, and A. A. Alonso. CRNreals: a toolbox for distinguishability and identifiability analysis of biochemical reaction networks. Bioinformatics, 28(11):1549-1550, June 2012. 
[27] G. Szederkényi. Computing sparse and dense realizations of reaction kinetic systems. Journal of Mathematical Chemistry, 47:551-568, 2010.

[28] G. Szederkényi, J. R. Banga, and A. A. Alonso. Inference of complex biological networks: distinguishability issues and optimization-based solutions. BMC Systems Biology, 5:177, 2011.

[29] G. Szederkényi, K. M. Hangos, and T. Péni. Maximal and minimal realizations of reaction kinetic systems: computation and properties. MATCH Commun. Math. Comput. Chem., 65:309-332, 2011.

[30] Y. Takeuchi. Global Dynamical Properties of Lotka-Volterra Systems. World Scientific, Singapore, 1996.

[31] P. Érdi and J. Tóth. Mathematical Models of Chemical Reactions. Theory and Applications of Deterministic and Stochastic Models. Manchester University Press, Princeton University Press, Manchester, Princeton, 1989. 